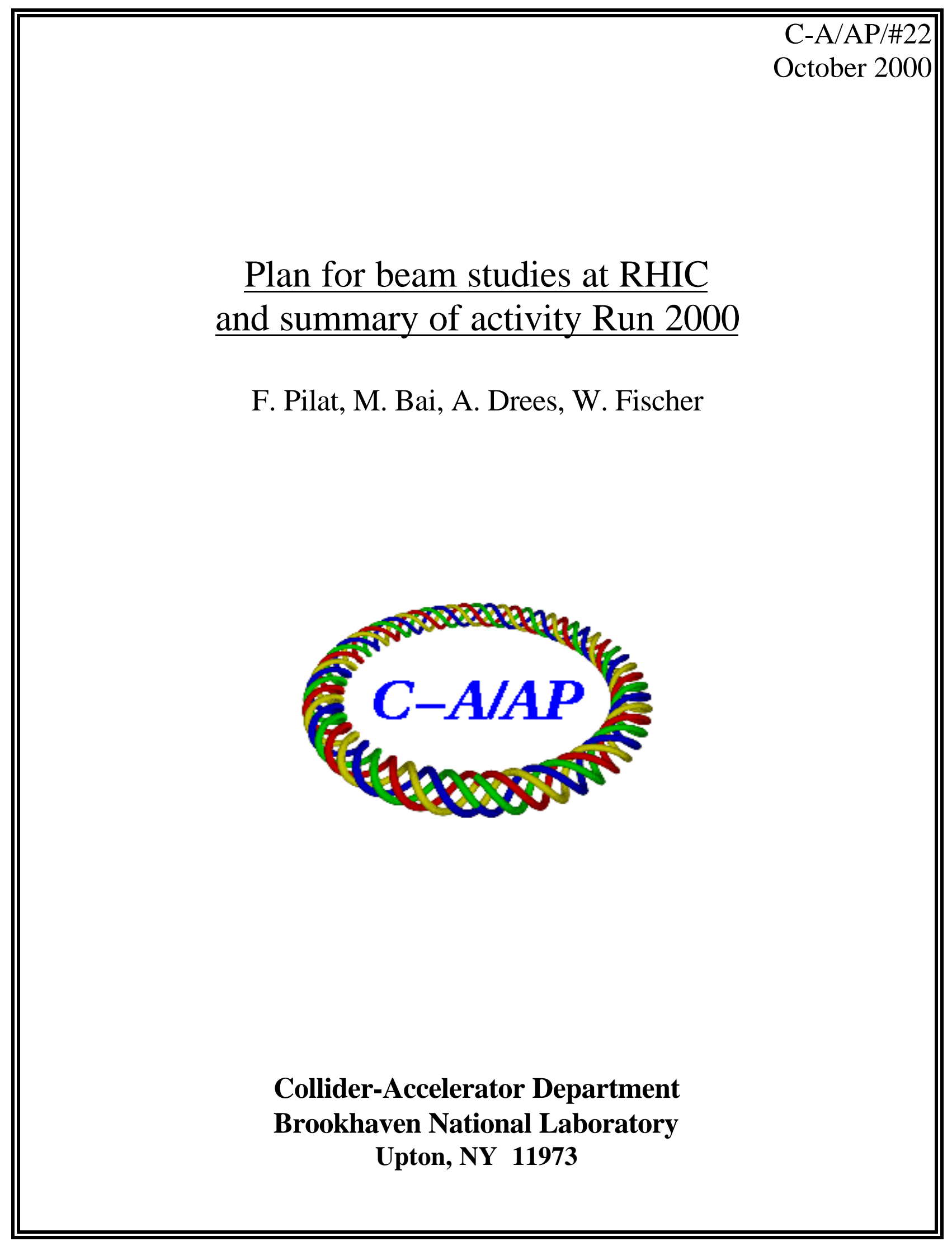




\title{
Plan for beam studies at RHIC and summary of activity during Run 2000.
}

\author{
F.Pilat, M.Bai, A.Drees, W. Fischer, \\ C-A Department, BNL
}

\section{Overview}

The aims of this document are (i) to summarize the plan for a program of initial beam studies at RHIC during the RHIC 2000 run, (ii) to give an overview of the beam studies that were carried out during the 2000 run and (iii) to initiate a discussion on beam studies to be carried out during the 2001 RHIC run.

An initial program of beam studies at RHIC emerged from discussions of operational needs and from discussions initiated at the workshop held at BNL in February 2000 on "Accelerator Physics Experiments for Future Hadron Collider", organized in the framework of the US-LHC Collaboration. Agenda, presentations and the Workshop Proceedings can be found on the Workshop WEB Page:

http://www.agsrhichome.bnl.gov/LHC/org/Beam2000/index.html.

The initial beam study program drafted at the Workshop eventually underwent a process of internal evaluation and development through meetings and discussions in within the C-A Department in the months preceding the start-up and during the early phase of beam operations in 2000 .

Run 2000 beam operations in RHIC started in earnest in April, and the focus between April and June has been the commissioning of the machine and the delivery of beam to the experimenters. With RHIC operations stable by August, culminating in the achievement of $10 \%$ of design luminosity, beam study activity started, as a natural evolution of commissioning activities. Beam studies have been largely parasitic, flexible, and integrated in shift activities.

What follows is the beam study plan in the areas that were identified as the best suited for an initial program, Interaction Regions studies (Section 2), Intra-Beam Scattering and Nonlinear measurements (Section 3), Experimental Background and Luminosity studies (Section 4), BeamBeam studies (Section 5) and studies with the AC Dipole (Section 6).

An overview of the beam studies activity during Run 2000 is presented in Section 7, with the understanding that the detailed discussion of the experimental findings will be the subject of individual papers or reports.

Finally, in Section 8, we start a discussion on beam study activities during the 2001 Run and possible evolution of beam experiment activity at RHIC in the years to come. 


\section{Interaction Region Studies (coordinator F.Pilat)}

\subsection{Goals}

The main goal of the IR correction system is to improve the performance of RHIC by:

(i) correcting locally coupling effects arising from field errors and misalignment in the IR triplets, DX and D0 magnets.

(ii) correcting locally the effect of the nonlinear field errors in the IR triplets, DX and D0 magnets.

Given the inherent complexity of nonlinear effects and the lack of straightforward observables, possible improvement of machine performance requires careful planning and machine studies to achieve the goal.

We will review the systems that are necessary for the IR Correction system, the modeling studies that guided the design of the system as well as modeling studies planned for the commissioning. The plan for studies is divided in a commissioning phase, in which equipment and methods are tested, and a study phase proper, in which parameter spaces are explored. Planning will have to reflect the realities and priorities of RHIC commissioning and operation.

\subsection{Systems}

The IR correction system consists of correction layers located in the $\mathrm{C} 1, \mathrm{C} 2$ and $\mathrm{C} 3$ corrector packages located next to the IR triplets, and related power supplies.

All IRs in the Blue and Yellow rings are equipped with correction layers, but in 2000 only layers at 6 and 8 o'clock are connected to 50A corrector supplies. A detailed layout of the IR regions can be found in the WEB page http://www.cadops.bnl.gov/AP/DryRun/ (link "Magnets and Power Supplies around 6 o'clock (8 o'clock)').

Other systems are necessary for IR Corrections:

Tune Meter. Tune measurements.

Schottky detector. Possible measurement of tune spread.

BPM's (turn-by-turn). Frequency analysis of turn-by-turn data to identify spectral lines due to nonlinear fields. For the 2000 run the capability exists of recording 128 turns at every BPM, and 10000 turns on selected channels.

Orbit Display. Display and correction of orbit. Setting up of IR bumps, off axis in the triplets to measure coupling locally by observing the off plane response and to measure effect of nonlinear fields.

DCCT. Measurement of beam current and beam lifetime. Real time (every $1 \mathrm{sec}$ ) monitoring and optimization of machine performance.

IPM. Profile measurements.

Kickers. To generate oscillations for turn-by-turn BPM acquisition, dynamic aperture measurements, etc. The tune meter kickers can be used resonantly. Should that not give a sufficient kick at collision, injection kickers can be used for vertical kicking. Abort kickers may be used to generate a horizontal kick at collision (in a low intensity machine run, and possibly only with a reduced number of kicker modules active)

AC Dipole. This is the ideal tool to generate a coherent oscillation for IR studies, and will be used for this purpose as soon as it is on line, likely in the 2001 RHIC run.

\subsection{Modeling}

A complete set of simulation results exists for the nominal RHIC collision lattice, $\left(\beta^{*}=1 \mathrm{~m}\right.$ at IP6 
and IP8, and 10m in all other IPs). The RHIC off-line model includes the measured field errors for all relevant magnets in the machine, measured at 5000A (closest to $100 \mathrm{GeV}$ ). The model includes also the "IR filter" that calculates the IR correction settings by the "action-kick" minimization procedure, and the local decoupling algorithm to set the IR skew quadrupole correctors operationally.

A model exists for the for the Run 2000 lattice at injection (feb2000a, $\beta^{*}=8 \mathrm{~m}$ at IP8 and IP10 and $\beta^{*}=3 \mathrm{~m}$ in all other IP's) and field errors at $540 \mathrm{~A}$, a current that is about $15 \%$ higher than the run 2000 injection current. A model exist for the Run 2000 storage lattice as well with field errors measured at $3000 \mathrm{~A}$ (closest current to $65 \mathrm{GeV}$ ). The model will need to be improved by extrapolating the measured field errors to the effective operating currents.

We also need to bridge the off-line to the on-line model by implementing in the latter the capability of reading and writing SXF files.

\subsection{System Commissioning}

The commissioning of the IR correction system consists of several steps. The prerequisite is to have one RHIC Ring (presumably Blue first, then Yellow) operational and stable. That means: stable circulating beam (lifetime $>1 \mathrm{~h}$ ), orbit corrected in the IRs to $<1 \mathrm{~mm}, \Delta \mathrm{Q}_{\min }<0.005$, and possibly one IP (IP6) squeezed to $1 \mathrm{~m}$. Commissioning with $\beta^{*}=3 \mathrm{~m}$ is of course possible but nonlinear effects would be lower.

\subsubsection{Nonlinear Correctors}

The plan is to test 1 layer at the time in the following order: normal sextupole (b2) normal octupole (b3) normal dodecapole(b5)

normal decapole (b4)

skew sextupole $(\mathrm{a} 2)$

skew octupole (a3)

skew dodecapole(a5)

(no power supply installed for run 2000)

(no power supply installed for run 2000)

For every layer:

1. Controlled experiment: apply corrector strength, measure effect on the machine (tune, orbit, lifetime, possibly spectral lines in turn-by-turn data), compare with model data.

Repeat that at positions of large $\beta_{\mathrm{x}}$ and $\beta_{\mathrm{y}}$ if we have 2 correctors in the same triplet (b3, b5).

2. Compensate the effect with a nearby corrector (for the b3 and b5 layers) or with correctors across the IP. Verify effect on the machine.

3. Set corrector at the value calculated by the "IR filter" to dead-reckon the measured field error.

4. Operational setting of the corrector based on machine observables (tune, DDCT, spectral lines). 5. Measurement of machine performance (lifetime, dynamic aperture) with and without correction.

\subsubsection{IR Coupling Correction System}

1. Measure IR coupling locally via IR bumps.

2. Controlled experiment: power one IR skew quadrupole and measure effect.

3. Compensate with IR skew quadrupole across the IR.

4. Set skew quadrupoles to value predicted by the IR filter and verify machine performance (2001) 
5. Measure coupling locally via turn-by-turn BPM data analysis (2001).

6. Correct coupling locally by local decoupling algorithm (2001).

\subsection{IR beam studies}

Once the IR correction system is commissioned there are several studies that can increase the knowledge and hopefully the performance of the machine, for instance:

1. Measure the effect of going off-axis in the triplets (Interaction Region bumps)

2. Parametric dependence on $\beta *$ at IP6, during a beta squeeze.

3. Effect of crossing angle (achievable in RHIC by trimming D0 and DX), to study interplay of IR field quality and beam-beam effects. That is particularly interesting with a proton beam where beam-beam effects are expected to be more significant. 


\section{IBS and Nonlinear Studies (coordinator W.Fischer)}

Initial studies of nonlinear and longitudinal effects will concentrate on effects that may limit the performance of RHIC now or in the near future. Proposed studies are:

3.1 Study of longitudinal growth due to intra-beam scattering

Variations in:

- Intensity

- Longitudinal focusing

- Longitudinal emittance

3.2 Measurement of transverse instability thresholds and growth rates

The goal is to estimate transverse damper requirements, with variations in:

- Intensity

- Fill pattern

- RF voltage

- Bunch length

- Transverse tunes

\subsection{Chromaticity measurement}

- from head-tail phase shift at injection

- from relative line width from LF Shottky spectra, as a function of tune at injection (persistent current decay)

3.4 Analysis of Schottky spectra to investigate the feasibility of stochastic cooling

3.5 Study of microwave instability below the transition energy 


\section{Experimental Background (Collimation) and Luminosity Studies (coordina- tor A.Drees)}

\subsection{Experimental background (Collimation)}

At RHIC one-stage collimators (scrapers) are installed to remove beam halo before it hits unprotected sections of the vacuum chamber (i.e. uncontrolled beam losses), damages experimental equipment or spoils the data quality. The collimators are designed for operation with gold particles. However, in one ring an additional collimator, a crystal, designed to improve the collimation efficiency for proton operations, is going to be installed about $8 \mathrm{~m}$ upstream of the scraper. Hence one two-stage collimation system will be available for beam studies next year. The crystal, about 5 $\mathrm{mm} \times 5 \mathrm{~mm}$ in size and movable in two planes, can be controlled to some $\mu \mathrm{m}$ precision in the horizontal plane and to sub-micron precision in the $\mathrm{x}-\mathrm{z}$-plane where its angle can be adjusted ( $\mathrm{z}$ corresponds to the direction of the beam). The following devices are available for steering, control and monitoring:

- An inch-worm, capable of sub-micron step movements for the angular motion.

- A stepping motor for horizontal alignment, allowing $\mu \mathrm{m}$ steps.

- A laser system to monitor the skew angle of the crystal to $\mu$ rad precision.

- LVDT's to monitor the horizontal and skew motion of the crystal.

- A phosphorus screen on the scraper edge, combined with a CCD camera for readout.

- About 365 standard RHIC beam loss monitors (BLM) around the two rings.

- 4 additional BLM's downstream of scraper, 2 on top and 2 at the bottom of the pipe.

- 4 PIN diode loss monitors (PD), surrounding the beam pipe downstream of scraper.

- Additional 8 PD's upstream of the scraper, evenly distributed around the beam pipe.

- A hodoscope next to the crystal to monitor particles scattering from its location.

We propose the following studies:

1. Study the scraper performance with both gold and proton beam

2. Study the performance of the crystal with gold and proton beam

3. Use the scrapers and/or the crystal to study IBS and beam growth rates (diffusion)

4. Experimental background as function of collimation scheme and beam parameters

\subsubsection{Scraper performance}

Measurement of the collimation efficiency and loss distribution around the ring

Study of the loss pattern dependence on particle type, intensities and scraper position.

Conditions: 1 bunch per beam, gold or protons, various beam intensities, collision energy.

\subsubsection{Crystal performance}

Measure of the collimation efficiency and loss distribution with crystal.

Comparison with results from using scrapers only.

Observation of channeling (phosphor screen).

Study of loss patterns depending on crystal positions, scraper positions and beam intensities.

Conditions: 1 bunch in yellow ring, proton and gold beam, various intensities, collision energy.

\subsubsection{Beam growth rate}

Use of the scraper and of the crystal (after sufficient experience with its performance is gained) to 
study beam growth rates due to intra beam scattering and diffusion, with and without collisions. Measure of loss rates as a function of time after fast (i.e. 1 second) retraction of the crystal.

Measure of growth rate dependence on beam intensities and particle type.

Conditions: 1 bunch in yellow ring, proton and gold beam, various intensities, storage energy.

\subsubsection{Experimental background rates}

Measure of the background rates seen by the experiments as a function of beam intensity, beam size, scraper position and debunched beam intensity.

Conditions: gold beam, store energy, large number of bunches, different intensities

\subsection{Luminosity Studies}

Collision rates are measured by the experimental detectors and by the ZDC's (Zero Degree Calorimeters) which are installed in all 4 experimental areas. Located between the DX and D0 magnets, they are well suited to measure Au-Au collision rates by detecting forward emitted neutrons from the collision point. Equipped with identical readout electronics in the 4 interaction regions, they provide comparable signals from all experiments.

\subsubsection{Neutron cross section}

Since the delivered luminosity is the key to machine performance it is essential to know the absolute cross section of the neutron emitting process. This cross section can be measured using the Vernier scan technique, where the two beams are scanned across each other while measuring collision rates.

\subsubsection{Total beam size}

Using the same Vernier scan technique, one can also measure the total transverse beam size of the blue and yellow rings together, at the beginning and at the end of a store. The Vernier scan data on growth rates can be compared with the reconstructed vertex positions from the experimental detectors as a function of time.

\subsubsection{Luminosity}

Comparison of measured luminosity and luminosity derived from beam parameters

Conditions: Gold beam only, collision energy, high number of bunches, high intensity 


\section{Beam-Beam studies (coordinator W.Fischer)}

With two beams of the same intensity RHIC is suited for the investigation of strong-strong beambeam effects. However, in the initial operation phase of RHIC we will concentrate our experimental efforts on a few problems only:

\subsection{Measurement of Beam-Beam Tune Shifts}

Detuning is an essential property of a nonlinear system. Models with incorrect tune shifts cannot be expected to reproduce or predict experimental results. For RHIC we expect the following tune shifts from beam-beam interactions:

\begin{tabular}{||c|c|c|c||}
\hline Species & $\begin{array}{c}\text { Intensity } \\
\text { [particles/bunch }]\end{array}$ & $\begin{array}{c}\text { Nrm. emittance } \\
{[\mathrm{mm} \text { mrad }]}\end{array}$ & $\Delta \mathrm{Q} / \mathrm{IR}$ \\
\hline \hline Gold & $1.0010^{9}$ & 10 & 0.0023 \\
\hline Gold & $0.5010^{8}$ & 10 & 0.0012 \\
\hline Gold & $1.0910^{9}$ & 20 & 0.0012 \\
\hline Protons & $1.0010^{11}$ & 20 & 0.0037 \\
\hline Protons & $0.5010^{11}$ & 20 & 0.0018 \\
\hline Protons & $1.0010^{11}$ & 40 & 0.0018 \\
\hline
\end{tabular}

\subsubsection{Coherent Beam-beam Tune Shift}

The coherent tune shift can be measured by changing the beam separation while observing the tune in the two beams. Measurements at collision energy provide a maximum separation value. Measurements can also be done with different intensities in the two beams.

Best experimental conditions:

\begin{tabular}{|l|l|}
\hline Species: & Protons \\
\hline Energy & Collision \\
\hline Number of Bunches: & 1 per beam \\
\hline Intensity: & $110^{11}$ protons per bunch \\
\hline
\end{tabular}

Round gaussian beams of the same intensity and head-on collisions are assumed in the 2 rings.

\subsubsection{Tune Spread due to Beam-Beam Interaction}

Once fully commissioned, the RHIC Schottky monitor can measure tune spreads of gold beams in a non-destructive manner. Tune spreads of beams that are not in collision can be compared with beams in collision to identify the tune spread attributable to beam-beam interaction.

Since the tune spread is recorded as a function of beam separation, the experiment should be done at storage energy to maximize the possible transverse separation value. 
Best experimental conditions:

\begin{tabular}{|l|l|}
\hline Species: & Gold \\
\hline Energy & Collision \\
\hline Number of Bunches: & 1 per beam \\
\hline Intensity: & $510^{8}$ ions per bunch \\
\hline
\end{tabular}

\subsection{Measurements of Collective Modes}

With two bunches of the same intensity collective transverse oscillation modes may be observable. An analysis of the conditions under which collective modes are present can be done by varying parameters as the intensity ratio of the two beams or the nonlinear tune shift due to octupoles. These conditions are currently being studied, primarily by M. Furman at LBL.

Best experimental conditions:

\begin{tabular}{||l|l|}
\hline Species: & Protons \\
\hline Energy & Collision \\
\hline Number of Bunches: & 1 per beam \\
\hline Intensity: & $110^{11}$ protons per bunch \\
\hline
\end{tabular}




\section{Studies with the AC Dipole (coordinator M.Bai)}

Beam dynamic experiments and machine studies often require a long coherent oscillation. The technique of using an AC dipole to adiabatically excite a sustained coherent oscillation without emittance growth has been successfully used in the AGS polarized proton acceleration experiment. Two AC dipole magnets with horizontal and vertical magnetic fields are under development for spin manipulations and beam dynamic studies at RHIC. Both magnets are about $1 \mathrm{~m}$ long and will be installed in the interaction region of sector 3 . The two magnets are designed to be able to induce a large coherent oscillation for the beam studies. In addition, the AC dipole with horizontal magnetic field can also be operated at the vicinity of the polarized proton spin precession frequency to manipulate the spin motion for the RHIC spin physics experiments.

The two magnets and their systems are still under construction and are expected to be available for the year 2001 beam run. For the year 2001 run, we would like to propose the following experiments.

\subsection{Measurement of the betatron functions and phase advance}

This measurement requires a coherent oscillation of a few $\sigma$ amplitude. Here, $\sigma$ is the rms beam size. The betatron function and phase advance between two BPMs can be retrieved from the turnby-turn data of the two BPMs, provided the AC dipole itself is not located between the 2 monitors.

\subsection{Measurement of the non-linear detuning}

In the presence of detuning with amplitude, two islands can be formed when the AC dipole frequency is beyond the bifurcation point. The two fixed points of the islands are determined by the detuning coefficient $\alpha$, where $\alpha=\mathrm{d} v / \mathrm{dJ}$. Here $v$ is the betatron tune and $\mathrm{J}$ is the action. The coefficient $\alpha$ can be measured by ramping the AC dipole frequency through the resonance and measuring the beam response function.

\subsection{Measurement of the higher order multipole components}

The non-linearity in the machine causes the phase space to be distorted. By exciting a coherent oscillation with a large amplitude, one can determine the multipole components from the spectrum of the turn-by-turn oscillation amplitude.

\subsection{Spin flip for RHIC spin physics}

In RHIC, the spin tune can be varied from $1 / 2$ by tuning the two snakes' axis. The AC dipole then induces an artificial spin resonance at its oscillating frequency. By ramping the AC dipole frequency through the spin precession frequency, a full spin flip can be induced. This will allow the RHIC spin physics experiment to have collisions with different bunch polarization patterns to reduce the systematic error. 


\section{Summary of beam study activity during Run 2000}

After cool-down, testing of power supplies and setup of the injectors, Run 2000 beam operations in RHIC started at the beginning of April. The first months were fully dedicated to the machine commissioning, with the achievement of first collisions on June 12th.

Table 1: Summary of beam studies for RHIC Run 2000

\begin{tabular}{|c|c|c|}
\hline Study Area & $\begin{array}{c}\text { Beam studies Run } 2000 \\
\text { with gold ions }\end{array}$ & $\begin{array}{c}\text { (Planned) beam studies } \\
\text { with protons }\end{array}$ \\
\hline $\begin{array}{l}\text { NONLINEAR/ } \\
\text { IBS }\end{array}$ & nonlinear detuning (inj) & beam profile \\
\hline (Fischer Tepikian Schmidt, & chromaticity vs. time (inj, sto) & dynamic aperture (inj) \\
\hline \multirow[t]{2}{*}{ Cameron) } & long. profile vs. time (inj, sto) & \\
\hline & IBS integrated Blue (inj) & IBS integrated (inj) \\
\hline $\begin{array}{l}\text { INTERACTION } \\
\text { REGIONS }\end{array}$ & IR bumps studies (inj) & IR bumps studies (inj) IP10 \\
\hline (Pilat, Ptitsyn, Koutchouk, & Blue: IP2 IP6 IP8 & IR bumps studies (sto) \\
\hline \multirow[t]{2}{*}{ Sen, Cardona) } & Yellow: IP2 IP6 IP8 & beta squeeze in IP6 \\
\hline & local decoupling (inj) IP8 & local decoupling (store) \\
\hline $\begin{array}{l}\text { COLLIMATION/ } \\
\text { LUMINOSITY }\end{array}$ & collimator performance (sto) & collimator performance (sto) \\
\hline (Drees, Fliller) & Vernier scans & measure of growth rates \\
\hline BEAM-BEAM & beam-beam tune shift (sto) & beam-beam tune shift (sto) \\
\hline \multicolumn{3}{|l|}{ (Fischer, Cameron) } \\
\hline MACHINE OPTICS & TBT orbit + kick (sto) Blue & TBT orbit + kick (sto) Yellow \\
\hline (Satogata, Bai, Drees) & dispersion (sto) Blue Yellow & \\
\hline
\end{tabular}

June and July saw improvements in machine performance, with an increased ramp rate, an almost 10 -fold increase in the number of bunches and an increase in bunch current. The increased reproducibility and reliability of the machine marked the transition between the commissioning of RHIC 
and operations for luminosity. With the goal of $10 \%$ of design luminosity for gold operations achieved and the experiments taking steadily data, the opportunity opened in August to devote a small fraction of machine time to beam studies.

Beam study activities for RHIC Run 2000, as planned during the BNL February Workshop and later, has focused on studies that are a natural follow up and extension of commissioning activities. Most of the study time was integrated into the daily shifts or parasitic, and typically carried out by small groups of BNL people, and in some cases with collaborators from outside institutions.

Table 1 summarizes the studies carried out during Run 2000 and studies that were initially planned for the 2 weeks of proton beam operations in September in the Yellow ring, in parallel with the polarized protons test run in the Blue ring. The Yellow ring was unfortunately taken offline by a water leak that spoiled the vacuum and with it the opportunity of using the Yellow beam for studies. The detailed description and analysis of the studies listed in Table 1 will be the content of upcoming individual papers and will not be discussed here.

\section{Conclusions and plans for Run 2001 and beyond.}

A beam study program was planned for the RHIC Year 2000 run and initial beam studies were carried out at RHIC in August and September 2000, mostly during gold operations.

The beam studies this year focused mainly on improvements of RHIC performance and on activities that are a natural extension of beam commissioning. The studies were typically carried out by small teams of RHIC beam commissioners and in some instances together with collaborators from outside institutions. Typically studies were integrated in the daily shifts, using beam parasitically or a few hours of dedicated beam studies.

The plan for beam studies presented in Sections 2-5 can be a basis from which to plan beam studies activities in the next run, likely to start in March 2001. Ideas will be reviewed at the RHIC Retreat in November 2000 and a more precise plan formed in the ensuing months. With RHIC being operational and with stringent requirements on luminosity coming from the experiments, the beam studies necessary to improve machine performance will benefit from the set up of Machine Development time, scheduled and agreed upon in advance with operations and the experiments.

In the longer run, from 2002 and beyond, RHIC will be into routine operations. At that time, larger, more formal collaborative experiments, dedicated to the understanding of issues of relevance to future hadron colliders (LHC, VLHC, etc.) will be proposed for approval to the laboratory, thus exploiting the full potential of RHIC. 